\title{
The Connection of Teaching and Computer to Improve the Teaching Quality
}

\author{
Shangwu Yang \\ Zhengzhou Tourism College, Yu ying lu 1th, Zhengzhou, 450009, China \\ yswhenan@yeah.net
}

\begin{abstract}
Computer is one of tools which have the great impact on human. In fact, it was found everywhere and at all times. It shortens the distance of people and becomes a part of people's life. It also has same great help to teaching. Computer assisted instruction (CAI) has an importance on modern teaching methods and it is also an effective one. In this article we consider the interactive activities between the computer and teaching activities. On the one hand is the theoretical link between the teaching and the computer. On the other hand is the computer's application on teaching. After the discussion we draw the conclusion that the CAI has an effective aid on teaching, especially in advanced mathematics. By considering we can do a better teaching job, it is not only the students can gain more, but also teachers can improve the teaching level from more breadth and depth of access to the quality of teaching.
\end{abstract}

Index Terms - Computer, Computer Assisted Instruction (CAI), Heuristic teaching.

\section{Introduction}

Computer can be said to have become an important part of our lives. It always affects our lives. We use it calculate goods' value at the supermarket; in airports, stations and other transport occasion it provides us with updated traffic information at any time; more magically, not to mention the Internet, on-line shopping, news browsing, on-line interactive etc, those cannot lack of the computers' assistance. As teachers, we are more concerned about how to use the computer well in the classroom, to improve the efficiency of classroom instruction, such that students can be gained the knowledge more effectively in the transient classroom's teaching activities.

Traditional teaching model has its limitations. In the traditional teaching the teacher is the domination of teaching activities. Teachers mainly determine the students' learning content and learning progress leading to students' learning initiative has been constrained. Now, we find in the teaching arrangements of the tutor time, less and less students participating the tutor course. On the contrary some students encounter puzzled problems in the after-school reviewing, but they can not immediately discuss with the teacher, perhaps the problem will be forgotten till the next class time. At the same time where students suffers puzzled point teachers can not go back and forth unlimited explain to meet the needs of students. Now more and more teaching activities add in the computer assisted instruction because of its unique teaching effectiveness: people use the computer's effects of interactive, multimedia features, guide, tools and transmission effects to develop the CAI Courseware. It can create the illustrations, vivid descriptions of the teaching's environment, stimulate learning enthusiasm and initiative of students which improve learning efficiency. The interaction of CAI is particularly suitable for inventing suitable individualized learning environment. According to the characteristics of different teaching schedules teachers can exploit teaching assistant system, learning support systems, laboratory support system, answering support system, practice support systems, test systems and other auxiliary systems. Using these systems students can be aware of the relationship among knowledge point and formation of knowledge structure. If they suffer the confused knowledge point then they can repeat finding the answer in the CAI courseware. It is impossible in tradition teaching model which students can dominate their time to study the progress.

As the CAI has become to a focus topic we consider the issue which is meaningful. We want to find the relation between the teaching and computer, where we consider this emphasis on interactive teaching and computer problems (mainly in advanced mathematics).It is not only to consider the computer how to help teaching more exciting, but also to make students understand computer theoretical basis better and more effective understanding of course materials. We want to make students understand advanced mathematics having a profound theoretical background of the computer by teaching.

\section{Theoretical Link between the Teaching and Computer}

If we want students to make better use of computers as an assistant, then teachers should explain the computer's basic theory to the students in the teaching activities. We consider the following aspects:

First, it is important to study the computer professional courses, which is the best link way to computer. As tools for the computer's connectivity, computer languages and algorithms are essential. From the initial BASIC, C-language to the current module language, we only learn computer science courses to better application of computer, while it can be played a larger role.

Second, learning other courses of the curriculum is also important to apply for the computer. As this context, most people lose sight of its role, but this is precisely its role can not be ignored. If we want to convince the students to connection between the courses and the computer, then we must find the juncture of them. For example, in advanced mathematics teaching, we all know computers and mathematics are inseparable. But to make us a detailed description of the 
specific combination of parts between them, it is not easy thing to explain.

In the Power Series in advanced mathematics' teaching section, we consider finding the corresponding Sum Function of the power series and the function's role is very clear: it is well known that it is difficulty to solve the sum of infinite power series. But if we find the sum function of the power series, then the value of power series is equals to the value of the sum function.

For example: We want to solve the sum of the following power series $1, \frac{1}{2}, \frac{1}{4}, \frac{1}{8}, \cdots, \frac{1}{2^{n}}, \cdots$

It is an infinite power series; we have no formula to solve the sum. So we can consider the finite sum of part of the power series, like to solve the forth m-th's sum. It is an easy thing since the power' property such that

$$
S_{m}=1+\frac{1}{2}+\frac{1}{4}+\cdots+\frac{1}{2^{m}}=\frac{1-\frac{1}{2^{m}}}{1-\frac{1}{2}}
$$

If we add the value of $\mathrm{m}$, then the part sum is closer the sum of power series. By the definition of limitation, we know that the part sum's limit is the sum of power series, denoted by S.

Therefore we have that

$$
S=\lim _{m \rightarrow \infty} S_{m}=\lim _{m \rightarrow \infty} \frac{1-\frac{1}{2^{m}}}{1-\frac{1}{2}}=2 .
$$

It is easy to calculate the value of sum function on the determined number. In the next section, we consider the function of the power series' Expansion. Normally, we consider it is an inverse problem of the above problem.

For example we want to the power series of a function, like the $\frac{1}{1-3 x}$.

It is hard to read the function for computer even it is so easy to us, since the basic compute ring rule of the computer. The computer is easy to read the power series so we have to send the power series of a function to computer.

$$
\text { Since } \frac{1}{1-3 x}=1+3 x+(3 x)^{2}+(3 x)^{3}+\cdots+(3 x)^{m}+\cdots, \quad(|3 x|<1)
$$

by the expansion of function. The expansion of a function is difficulty to solve, but it provides a style of the connection between man and computer.

It is not deep-seated the problem. Why do this inverse problem? Because it in order to meet the needs of the computer. From the principle of the computer we know it only has addition calculation. We let a function unfold the power series form to communicate with the basic theory of the computer. If we explain this issue to the students, they can understand the advanced mathematics' application better. On the contrary, it is also an inspiration of teachers. It stimulates teachers to explore the information and more profound knowledge in the advanced mathematics. At the same time the teachers can raise their teaching level. Raising the level of teaching gives the teachers more self-confidence and stimulates students' interest to learn more.

\section{The Computer's Application on Teaching}

For the application of computers in teaching, many scholars have discussed. We only consider its application on advanced mathematics.

First, the computer is widely used in teaching and it can be more closely integrate abstract thinking and thinking in images. To explain this action we explore the display of function graphics coming from the advanced mathematics.

1. It is well known that the thought of combination the function with graphics is shaped by solving the correspondence and mutual aid of the function and graphics. The difficulty of the thought is in finding out internal connection and achieving conversion of abstract conception and graphics. It is evident that computer is effective to simulate the fixed point trajectory and reveal dynamic relationship of the function and graphics, which can provide the students' abstract thinking a vivid image of the specific situation. Many graphics are difficulty to draw out by hand in advanced mathematics, like in the multidimensional space of graphics such as saddle surface, etc. Students are not easy to imagine the graph structure. But to understand the spatial structure of graphics is very important to understand the following abstract concept. Another example is the limit problem for the multi-function. For the students' understanding multi-function graphics is an importance to the trend function. If they can have the imagine of the graphical structure, then they can find out the tread function and understand the concept of multi-function limit.

2. The combination is also reflected in the "mathematical experiment" in CAI. The "mathematical experiment" is adopted by means of experiment such that students can learn more intuitive understanding of mathematical knowledge. A lot of the "mathematical experiment" tools, such as "Geometer's Sketchpad", "Mathtool" and other excellent tools, provide students to experience the essence of the advanced mathematics and to play an unlimited imagination by doing the experiment. Like the Polar Equation of the curve, most students are not taught in the secondary school. However it is an important tool in advanced mathematics. They can not be familiar with the curve's representation in polar coordinates or have a clear imagine of its trajectory. But the mathematical software can show the trajectory of the curve. Like $\rho=2 a \cos \theta$, its trajectory is a circle. Let students do the experiment $\rho=a(1+\cos \theta)$. The graphics is a "Peach Line". They are familiar with this signal, which can stimulate their curiosities and further consolidate the combination of function and graphics.

Second, it is applied in the approximate calculation. In reality many problems we encountered are concerned about finding the solution of approximate calculation. The common 
methods are consisting of Iteration tools. During the iteration we need solve the calculation problems. This is the superiority of the computer and some good software supporting it, like matlab etc. In the advanced mathematics there is a problem about finding the rule of the known data, i. e., finding a approximate graphics of the data's trajectory. The involved method includes "Newton" interpolation method, data mining and so on. These all need the computer's support. And the computer can display the according graphics intuitively.

Third, it improves the students' learning ability. Many scholars have considered "mission-driven method", i. e., the teacher arranges the learning tasks to students and let they find the solutions of the tasks in CAI. For example in Line Programming, students can analysis the task and build the according LP model. There are many algorithms to solve the LP problem, like Dijkstra Algorithm etc. The computer can display the optimal solution to the objective function using the algorithms' software. This can improve student achievement feeling and the abilities of solving practical problems.

\section{Conclusions}

The word "data" is plural, not singular. In American Computer-aided teaching is a new form of teaching model. It has changed the traditional teaching mode, making classroom teaching more colorful and stimulating students' interest in learning by the computer multimedia features. How to use the computer-aided instruction well, it is the current focus topics of teaching. Different scholars have different views. Although a lot of controversy, most academics are certain on the emergence of CAI. We consider this issue on advanced mathematics and draw a conclusion that it is an effective teaching aid tool. It makes the combination of abstract thinking and thinking in images. Students can understand the link of the function's graphics and the corresponding mathematical concepts (limits, etc). Teachers can use the "task-driven method" to active students' participation in teaching activities, and use "mathematical experiments" to improve students' practical ability. Based on the above analysis, we should have efforts to analysis of teaching materials, improve the class teaching's efficiency by helping of CAI and help students build good habits of study and thinking way.

Finally, we have an data to analysis of using the CAI improving the study of interesting of students', the data from the volunteers of students and the number is

Table 1. An example of a CAI' data

\begin{tabular}{|c|c|c|}
\hline An example of a column heading & $\begin{array}{c}\text { The former } \\
\text { percent }\end{array}$ & $\begin{array}{c}\text { Using the CAI' } \\
\text { percent }\end{array}$ \\
\hline Feeling the intersting of study & 60 & 80 \\
\hline Knowing the abstact knowledge & 45 & 75 \\
\hline Finding the value of knowledge & 20 & 68 \\
\hline Improving the practice ability & 35 & 70 \\
\hline $\begin{array}{c}\text { Knowing how to find the connection of } \\
\text { different things }\end{array}$ & 10 & 65 \\
\hline
\end{tabular}

From the CAI'data we know the assistant of computer is useful to the class teaching. We still should find the new orientation of using the computer' assisting to improving the teaching level, although there are many aspects needed us to consider.

\section{References}

[1] Zhang Ju, "Heuristic teaching," Journal of Chifeng University(Natural Science Edition)Systems Engineering and Electronic, vol. 23, no. 2, pp. 56-57, 2001.

[2] Lin Qinjie, Qing Huiyang, Basic Computer Course, Changsha: National University of Defiance Technology Press, 2008, pp.5-30.

[3] Wang Zixi, Jiao Yue, and $\mathrm{Hu}$ Jinsong, "Methodology to Improve Effectiveness of Educations for Computer Application Courses," Computer Knowledge And Technology, vol. 4, no. 5, pp. 1156-1157, 2008.

[4] Y. Yorozu, M. Hirano, K. Oka, and Y. Tagawa, "Electron spectroscopy studies on magneto-optical media and plastic substrate interface," IEEE Translated J. Magn. Japan, vol. 2, pp. 740-741, August 1987 [Digest $9^{\text {th }}$ Annual Conf. Magnetics Japan, p. 301, 1982]. 